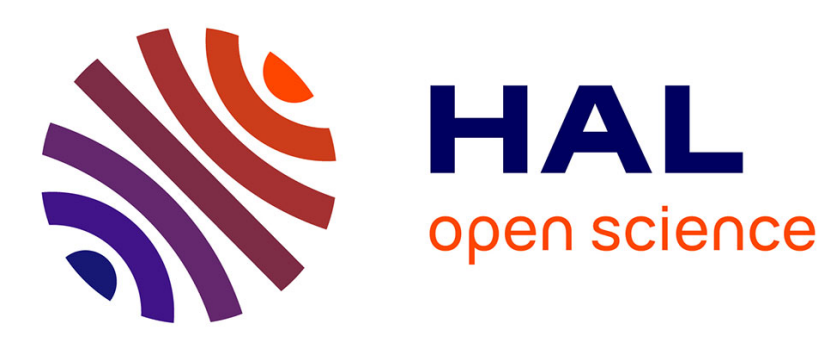

\title{
Finding Approximate and Constrained Motifs in Graphs
}

Riccardo Dondi, Guillaume Fertin, Stéphane Vialette

\section{To cite this version:}

Riccardo Dondi, Guillaume Fertin, Stéphane Vialette. Finding Approximate and Constrained Motifs in Graphs. CPM 2011, 2011, Palermo, Italy. pp.388-401, 10.1007/978-3-642-21458-5_33 . hal00606173

\section{HAL Id: hal-00606173 \\ https://hal.science/hal-00606173}

Submitted on 5 Jul 2011

HAL is a multi-disciplinary open access archive for the deposit and dissemination of scientific research documents, whether they are published or not. The documents may come from teaching and research institutions in France or abroad, or from public or private research centers.
L'archive ouverte pluridisciplinaire HAL, est destinée au dépôt et à la diffusion de documents scientifiques de niveau recherche, publiés ou non, émanant des établissements d'enseignement et de recherche français ou étrangers, des laboratoires publics ou privés. 


\title{
Finding Approximate and Constrained Motifs in Graphs
}

\author{
Riccardo Dondi ${ }^{1}$, Guillaume Fertin ${ }^{2}$, and Stéphane Vialette ${ }^{3}$ \\ 1 Dipartimento di Scienze dei Linguaggi, della Comunicazione e degli Studi Culturali \\ Università degli Studi di Bergamo, Via Donizetti 3, 24129 Bergamo - Italy \\ riccardo.dondi@unimib.it \\ 2 Laboratoire d'Informatique de Nantes-Atlantique (LINA), UMR CNRS 6241 \\ Université de Nantes, 2 rue de la Houssinière, 44322 Nantes Cedex 3 - France \\ guillaume.fertin@univ-nantes.fr \\ 3 IGM-LabInfo, CNRS UMR 8049, Université Paris-Est, \\ 5 Bd Descartes 77454 Marne-la-Vallée, France \\ vialette@univ-mlv.fr
}

\begin{abstract}
One of the emerging topics in the analysis of biological networks is the inference of motifs inside a network. In the context of metabolic network analysis, a recent approach introduced in [14], represents the network as a vertex-colored graph, while a motif $\mathcal{M}$ is represented as a multiset of colors. An occurrence of a motif $\mathcal{M}$ in a vertexcolored graph $G$ is a connected induced subgraph of $G$ whose vertex set is colored exactly as $\mathcal{M}$. We investigate three different variants of the initial problem. The first two variants, Min-AdD and Min-Substitute, deal with approximate occurrences of a motif in the graph, while the third variant, Constrained Graph Motif (or CGM for short), constrains the motif to contain a given set of vertices. We investigate the classical and parameterized complexity of the three problems. We show that MiNAdD and Min-Substitute are NP-hard, even when $\mathcal{M}$ is a set, and the graph is a tree of degree bounded by 4 in which each color appears at most twice. Moreover, we show that Min-Substitute is in FPT when parameterized by the size of $\mathcal{M}$. Finally, we consider the parameterized complexity of the CGM problem, and we give a fixed-parameter algorithm for graphs of bounded treewidth, while we show that the problem is $W[2]$-hard, even if the input graph has diameter 2 .
\end{abstract}

\section{Introduction}

The problem of analyzing biological networks such as protein-protein interaction networks and metabolic networks has become increasingly relevant in Computational Biology (see for example $[5,12,13,17-19])$. While the classical approach is based on graph-theoretical topology of the motif, a recent approach introduced in [14] aims at discovering functional motifs that do not rely on the conservation of the topology, but that are simply connected components of the network. This approach has been formalized as a graph problem (named GRAPH MOTIF), in which given a vertex-colored graph $G=(V, E)$ and a multiset $\mathcal{M}$ of colors, the 
goal is to find a subset $V^{\prime} \subseteq V$ which is connected and whose vertex set is colored exactly as $\mathcal{M}$.

The Graph Motif problem has been widely studied, and some variants have been introduced. The original problem is known to be NP-complete [14], even if the input graph is a tree with maximum degree 3 and the motif is a set [10], and if the input graph is a bipartite graph with maximum degree 4 and the motif is built over two colors only [10]. It is easy to see that GRAPH Motif admits a polynomial time algorithm when the input graph is a tree and each color occurs at most twice in the input tree. The GRAPH MotIF problem is known to be in FPT, when parameterized by the size of the motif $[4,10,11]$, while it is W[1]-hard when parameterized by the number of distinct colors in the motif, even in the case the input graph is a tree [10]. Recently, the kernelization complexity of the problem has also been considered [1].

Different variants of the GRAPH MOTIF problem have been introduced. Such variants either modify the requirement of connectedness [7], or look for approximate occurrences of the motif, where some colors are allowed to be inserted or deleted in an occurrence of the motif $[5,8,11]$. Following this direction, we consider three variants of the GRAPH MoTIF problem. In the first two variants, we relax the constraint that each color of $\mathcal{M}$ must appear in an occurrence of the motif, and we allow for the adding (MIN-ADD) or the substitution (MINSubstitute) of some colors. These two problems are motivated by the fact that, due to experimental errors, there may not exist an exact occurrence of the motif $\mathcal{M}$ in the graph $G$. In the third variant, Constrained Graph Motif (or CGM, for short), we strengthen the requirement of connectedness, constraining some vertices of the input graph to be part of an occurrence of a motif $\mathcal{M}$. This is motivated by the fact that, due to a previous knowledge on the structure of the network, we may require some of the vertices to be contained in any occurrence of $\mathcal{M}$.

The rest of the paper is organized as follows. In Section 2, we give some preliminary definitions and we formally define the problems. In Section 3, we show that Min-Substitute and Min-AdD are NP-hard, even when $\mathcal{M}$ is a set, the input graph is a tree $T$ of degree bounded by 4 and each color has at most two occurrences in $T$. Notice that under the same hypotheses, the GRAPH Motif problem admits a polynomial time algorithm. In Section 4, we give an FPT algorithm for Min-SuBstituTE. In Section 5, we discuss the parameterized complexity of the CGM problem, when the parameter is the number of colors not belonging to mandatory vertices ; in Section 5.1, we show that CGM is fixed-parameter tractable for graphs of bounded treewidth, while in Section 5.2 we show that CGM is $W$ [2]-hard, even if the diameter of the input graph is bounded by 2 . Some of the proofs are omitted due to space constraints.

\section{Preliminaries}

In this section, we recall basic notations used in the rest of the paper. Given a graph $G=(V, E)$ and $V^{\prime} \subseteq V$, we denote by $G\left[V^{\prime}\right]$ the subgraph of $G$ induced 
by $V^{\prime}$, that is $G\left[V^{\prime}\right]=\left(V^{\prime}, E^{\prime}\right)$ and $\{u, v\} \in E^{\prime}$ iff $u, v \in V^{\prime}$ and $\{u, v\} \in E$. Given a vertex $v \in V$, we denote by $N(v)$ the set of vertices in $G$ adjacent to $v$. We recall that a graph is cubic when each vertex has degree 3 .

Let $G$ be a connected graph, where every vertex $u \in V(G)$ is assigned a color $c(u)$ from a set $\mathcal{C}$ of colors. For any subset $V^{\prime}$ of $V$, let $C\left(V^{\prime}\right)$ be the multiset of colors assigned to the vertices in $V^{\prime}$. Let $\mathcal{M}$ be a multiset of colors, whose colors are taken from the set $\mathcal{C}$. Given a colored graph $G$ and a subset of vertices $V^{\prime} \subseteq V(G), C\left(V^{\prime}\right)$ is said to match a multiset of colors $\mathcal{M}$ if $C\left(V^{\prime}\right)$ is equal to $\mathcal{M}$. In this case, by abuse of notation, we say that $V^{\prime}$ matches $\mathcal{M}$. Given a subset of vertices $V^{\prime} \subseteq V(G)$ such that $V^{\prime}$ matches $\mathcal{M}$ and $G\left[V^{\prime}\right]$ is connected, then $V^{\prime}$ is called an occurrence of $\mathcal{M}$ in $G$. A motif $\mathcal{M}$ is said colorful when $\mathcal{M}$ is a set of colors (rather than a multiset).

In this paper, we consider three variants of the GraPh Motif problem. For two of them, Min-Add and Min-Substitute, we look for a vertex set $V^{\prime}$ of $G=(V, E)$, such that $G\left[V^{\prime}\right]$ is connected and $C\left(V^{\prime}\right)$ is not necessarily equal to $\mathcal{M}$. Furthermore, we consider a constrained variant of the GRAPH MotiF problem, CGM, where the input consists of a vertex colored graph and a set of mandatory vertices that must belong to any occurrence of motif $\mathcal{M}$.

Let us introduce the first two variants of GRAPH Motif problem.

Min-ADD (decision version)

Input: A multiset of colors $\mathcal{M}$, a vertex-colored graph $G=(V, E)$, an integer $p$. Question: Is there a subset $V^{\prime} \subseteq V$, such that $G\left[V^{\prime}\right]$ is connected, $C\left(V^{\prime}\right) \supseteq \mathcal{M}$ and $\left|C\left(V^{\prime}\right) \backslash \mathcal{M}\right| \leq p$ ?

Min-SubStitute (decision version)

Input : A multiset of colors $\mathcal{M}$, a vertex-colored graph $G=(V, E)$, an integer $p$. Question : Is there a subset $V^{\prime} \subseteq V$, such that $G\left[V^{\prime}\right]$ is connected and $C\left(V^{\prime}\right)$ can be obtained with at most $p$ substitutions from $\mathcal{M}$ ?

Notice that, in case $p=0$, both Min-AdD and Min-Substitute are equivalent to the Graph Motif problem. As a consequence, Min-Add and MinSubstitute are both NP-hard when the motif is colorful, the input graph consists of a tree $T$ and each color has at most 3 occurrences in $T$ [10]. Furthermore, Min-AdD (resp. Min-Substitute) cannot be approximated within any approximation factor, and does not admit any fixed-parameter tractable algorithm, when the parameter is the number of added colors (resp. the number of substitutions). Notice that MIN-ADD is in FPT, when parameterized by $|\mathcal{M}|$. Indeed, in [11], a variant of Graph Motif, called Multiset Graph Motif With Gaps (MGMG), is considered: given an input graph $G$ and a motif $\mathcal{M}$, we look for an occurrence of $\mathcal{M}$ that is allowed to contain gaps. Note that this is precisely MIN-ADD, where the gaps represent colors to be added to $\mathcal{M}$. As in [11] it is shown that MGMG is in FPT when parameterized by $|\mathcal{M}|$, we can conclude that 
the MiN-ADD problem is in FPT. Furthermore, in case the motif is colorful, a fixed-parameter algorithm for MIN-ADD has been given in [5].

Let us now consider a different variant of the GRAPH MoTIF problem, called Constrained Graph Motif (CGM).

Constrained Graph Motif (CGM)

Input : A multiset of colors $\mathcal{M}$, a vertex-colored graph $G=(V, E)$, a set of mandatory vertices $V_{M} \subseteq V$.

Question: Is there a subset $V^{\prime} \subseteq V$, such that $G\left[V^{\prime}\right]$ is connected, $C\left(V^{\prime}\right)=\mathcal{M}$ and $V_{M} \subseteq V^{\prime}$ ?

Given an instance of CGM, define the optional occurrences $C_{o}$ as $C_{o}=\mathcal{M} \backslash$ $C\left(V_{M}\right)$.

The CGM problem is NP-complete, since the GRAPH Motif problem is NP-complete $[10,14]$. It is easy to see that CGM is fixed-parameter tractable, when the parameter is the size of the motif. Indeed, recall that GRAPH Motif is fixed-parameter tractable. By recoloring the graph, assigning a unique color to each vertex in $V_{M}$, and by modifying accordingly $\mathcal{M}$, we can conclude that each occurrence of $\mathcal{M}$ in $G$ must include all the vertices in $V_{M}$.

In Section 5, we investigate the parameterized complexity of the CGM problem, when the parameter is the number of optional occurrences. Notice that the Minimum (Unweighted) Steiner Tree problem is a restriction of the CGM problem, where the non mandatory vertices in the Steiner Tree problem correspond to optional occurrences in CGM. As the Minimum (Unweighted) Steiner Tree problem is W[2]-hard when parameterized by the number of non mandatory vertices [6], it follows that the CGM problem is $\mathrm{W}[2]$-hard when parameterized by the number of optional occurrences.

In Section 5.1, we will consider the case where the input graph has bounded treewidth and we will use a tree decomposition of the graph. Let us recall the definition of tree decomposition of a graph $[9,15]$. Given a graph $G=(V, E)$, a tree decomposition of $G$ is a pair $\left\langle\left\{X_{i}: i \in I\right\}, T\right\rangle$, such that each $X_{i}$ is called a $b a g$, and $T$ is a tree having as vertices the elements of $I$ and such that:

1. $\cup_{i \in I} X_{i}=V$;

2. for each edge $\{u, v\} \in E$, there is a bag $X_{i}$ with $u, v \in X_{i}$;

3. for each $i, j, k$ in $V$, if $j$ is on the path from $i$ to $k$ in $G$, then $X_{i} \cap X_{k} \subseteq X_{j}$.

The width of $\left\langle\left\{X_{i}: i \in I\right\}, T\right\rangle$ is equal to $\max \left\{\left|X_{i}\right|: i \in I\right\}-1$ and the treewidth of a graph $G$ is equal to the minimum $\delta$ such that $G$ has a tree decomposition of width $\delta$. A tree decomposition $\left\langle\left\{X_{i}, i \in\{1, \ldots, p\}\right\}, T\right\rangle$ of a graph $G$ is nice (see [15]) when, given a vertex $i$ of the tree decomposition, $i$ has at most two children and the following conditions hold:

1. if $i$ has two children $j$ and $k$, then $X_{i}=X_{j}=X_{k}$;

2. if $i$ has exactly one child $j$, then one of the following conditions holds:

(a) $\left|X_{i}\right|=\left|X_{j}\right|+1$, and then $X_{j} \subset X_{i}$; or 
(b) $\left|X_{i}\right|=\left|X_{j}\right|-1$, and then $X_{j} \supset X_{i}$.

In the rest of the paper, in order to extend some results from the case when $\mathcal{M}$ is colorful to the general case, we use the recoloring technique introduced in [4], based on the color-coding technique [3]. The recoloring technique starts from a general motif $\mathcal{M}$ and computes a colorful motif $C$, recoloring accordingly the vertices of the input graph $G$. Let $V^{\prime}$ be an occurrence of $\mathcal{M}$ in the graph $G$, then $V^{\prime}$ achieves a colorful recoloring if $C\left(V^{\prime}\right)$ is colorful after the recoloring of $\mathcal{M}$ and $G$. In [4], the following result was shown:

Lemma 1 (Betzler et al. [4]). Given a motif $\mathcal{M}$, the number of trials to achieve a colorful recoloring of $\mathcal{M}$ with an error probability of $\varepsilon$ is $|\ln (\varepsilon)|$. $O\left(e^{|\mathcal{M}|}\right)$.

\section{NP-hardness of Min-Substitute and Min-Add}

In this section, we show that Min-Substitute and Min-AdD are NP-hard, even if the input graph is a tree, the motif is colorful and each color has at most two occurrences in the input tree. Recall that, under the same hypotheses, the GRAPH MOTIF problem admits a polynomial time algorithm.

Theorem 1. The Min-SubstiTute problem is NP-hard, even when the input graph is a tree of maximum degree 4 , each color occurs at most twice in the input graph and the motif is colorful.

Proof. We give a reduction from the Minimum Vertex-Cover on Cubic Graphs problem (Min-VCC). Let $G=(V, E)$ be a cubic graph with $V=\left\{v_{1}, v_{2}, \ldots, v_{n}\right\}$, the Min-VCC problem asks for a subset $V^{\prime} \subseteq V$ of size at most $p$, such that for each $\left\{v_{i}, v_{j}\right\} \in E$ at least one of $v_{i}, v_{j}$ is in $V^{\prime}$. Min-VCC is known to be NP-hard [2]. Starting from $G$, we construct an instance of the Min-Substitute problem which consists of a tree $T$ and a set of colors $\mathcal{M}$. For any vertex $v_{i} \in V$, let $e_{i, j}, 1 \leq j \leq 3$, be its 3 incident edges, ordered arbitrarily. The tree $T=\left(V_{T}, E_{T}\right)$ is defined as follows (see Figure 1):

$-V_{T}=\left\{l_{i}, a_{i, 1}, a_{i, 2}: 1 \leq i \leq n\right\} \cup\left\{s_{i}: 1 \leq i \leq p\right\} \cup\left\{t_{i}: 1 \leq i \leq n+1\right\} \cup\left\{e_{i, j}:\right.$ $1 \leq i \leq n \wedge 1 \leq j \leq 3\}$

$-E_{T}=\left\{\left\{l_{i}, l_{i+1}\right\}: 1 \leq i<n\right\} \cup\left\{\left\{s_{i}, s_{i+1}\right\}: 1 \leq i<p\right\} \cup\left\{\left\{t_{i}, t_{i+1}\right\}:\right.$ $1 \leq i<n+1\} \cup\left\{\left\{l_{n}, t_{1}\right\}\right\} \cup\left\{\left\{t_{n+1}, s_{1}\right\}\right\} \cup\left\{\left\{l_{i}, a_{i, 1}\right\},\left\{a_{i, 1}, a_{i, 2}\right\}: 1 \leq i \leq\right.$ $n\} \cup\left\{\left\{a_{i, 2}, e_{i, j}\right\}: 1 \leq i \leq n \wedge 1 \leq j \leq 3\right\}$.

Clearly, this construction gives us a tree of maximum degree 4 . Let us describe the colors assigned to each vertex of $V(G)$. Each vertex $l_{i}, 1 \leq i \leq n$, is assigned a unique color $c\left(l_{i}\right)$, each vertex $s_{i}, 1 \leq i \leq p$, is assigned a unique color $c\left(s_{i}\right)$, and each each vertex $t_{i}, 1 \leq i \leq n+1$, is assigned a unique color $c\left(t_{i}\right)$. The two vertices $a_{i, 1}, a_{i, 2}, 1 \leq i \leq n$, are assigned the same color $c\left(v_{i}\right)$. Finally, each vertex $e_{i, x}$ in $V_{T}, 1 \leq i \leq n$ and $1 \leq x \leq 3$, associated to an edge $e_{i, j}=\left\{v_{i}, v_{j}\right\}$ 


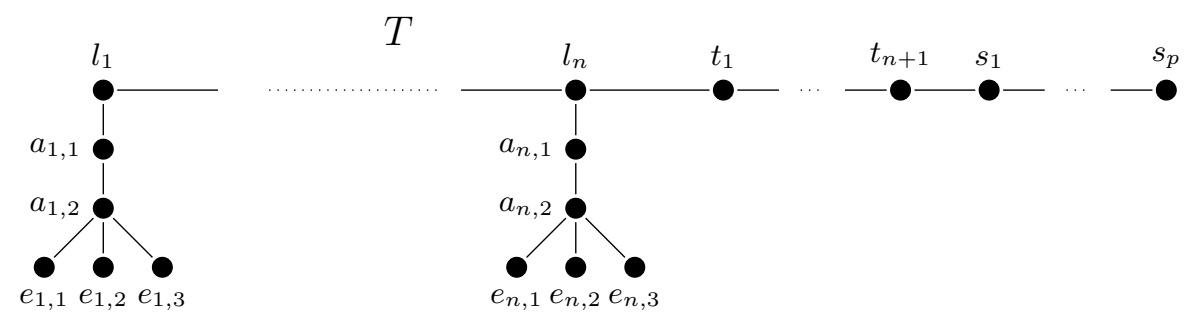

Fig. 1. Illustration of the reduction from Min-VCC to Min-Substitute.

in $E$, is assigned color $c\left(e_{i, j}\right)$. Each color occurs at most twice in $T$, as each color $c\left(e_{i, j}\right)$ is associated to two vertices of $T$, while each color $c\left(v_{i}\right)$ is associated to vertices $a_{i, 1}, a_{i, 2} . \mathcal{M}$ is a set of colors defined as follows: $\mathcal{M}=\left\{c\left(l_{i}\right): 1 \leq i \leq\right.$ $n\} \cup\left\{c\left(s_{i}\right): 1 \leq i \leq p\right\} \cup\left\{c\left(v_{i}\right): 1 \leq i \leq n\right\} \cup\left\{c\left(e_{i, j}\right): e_{i, j} \in E\right\}$. Notice that no occurrence of a color $c\left(t_{i}\right), 1 \leq i \leq n+1$, belongs to $\mathcal{M}$.

Starting from a vertex cover $V^{\prime} \subseteq V$ of $G$ of size at most $p$, a solution $V_{T^{\prime}}$ of Min-Substitute, that substitutes $p$ colors from $\mathcal{M}$, is obtained as follows. Given an edge $e_{i, j}=\left\{v_{i}, v_{j}\right\}$, define $e_{i, j}^{\min }=\min \{i, j\}$. The vertex set $V_{T^{\prime}}$ defined as follows:

$V_{T^{\prime}}=\left\{l_{i}, a_{i, 1}: 1 \leq i \leq n\right\} \cup\left\{t_{i}: 1 \leq i \leq p-\left|V^{\prime}\right|\right\} \cup\left\{a_{i, 2}: v_{i} \in V^{\prime}\right\} \cup\left\{e_{i, x}:\right.$ $\left.c\left(e_{i, x}\right)=c\left(e_{i, j}\right) \wedge i=e_{i, j}^{\min }\right\}$.

By construction and since $V^{\prime}$ is a vertex cover, $V_{T^{\prime}}$ induces a subtree of $T$. It is easy to see that, given $C\left(V_{T^{\prime}}\right)=\mathcal{M}^{\prime}, \mathcal{M}^{\prime}$ can be obtained from $\mathcal{M}$ by $p$ substitutions.

Let us consider now a solution $V_{T^{\prime}}$ of Min-Substitute, where $C\left(V_{T^{\prime}}\right)=\mathcal{M}^{\prime}$, $\left|\mathcal{M}^{\prime}\right|=|\mathcal{M}|$, and $\mathcal{M}^{\prime}$ can be obtained from $\mathcal{M}$ with at most $p$ substitutions. First, we show that $V_{T^{\prime}}$ does not contain a vertex of the set $\left\{s_{i}: 1 \leq i \leq p\right\}$. Indeed, assume that a vertex $s_{i}$ is part of $V_{T^{\prime}}$; by construction the set of vertices $\left\{t_{j}\right.$ : $1 \leq j \leq n+1\}$ must belong to $V_{T^{\prime}}$, and since $\mathcal{M}$ does not contain occurrences of any color $c\left(t_{j}\right), 1 \leq j \leq n+1$, it follows that $\mathcal{M}^{\prime}$ requires at least $n+1$ substitutions. Notice that $n+1>p$, as each vertex cover $V^{\prime}$ of $G$ has size at most $n$. Hence, we can assume that $V_{T^{\prime}}$ does not contain any vertex in the set $\left\{s_{i}: 1 \leq i \leq p\right\}$. It follows that all the colors $c\left(s_{i}\right), 1 \leq i \leq p$, in $\mathcal{M}$ must be substituted, and, since by hypothesis $\mathcal{M}^{\prime}$ can be obtained from $\mathcal{M}$ with at most $p$ substitutions, it follows that only the colors $c\left(s_{i}\right), 1 \leq i \leq p$, are substituted. Hence $\left\{l_{i}, a_{i, 1}: 1 \leq i \leq n\right\} \subseteq V_{T^{\prime}}$ and $\mathcal{M}^{\prime} \supseteq\left\{c\left(e_{i, j}\right): e_{i, j} \in E\right\}$. Since $T\left[V_{T^{\prime}}\right]$ must be connected, it follows that each vertex colored $c\left(e_{i, j}\right)$ must be connected to some vertex $a_{i, 2} \in V_{T^{\prime}}$ colored by $c\left(v_{i}\right)$. Define $V^{\prime}=\left\{v_{i}: a_{i, 2} \in V_{T^{\prime}}\right\}$; then $V^{\prime}$ is a cover of $G$ of size at most $p$, which completes the proof.

Theorem 2. The MIN-ADD problem is NP-hard, even when the input graph is a tree of maximum degree 4 , each color occurs at most twice in the input graph and the motif is colorful. 
Proof. (Sketch) The result follows from a reduction from Min-VCC similar to that of Theorem 1. Given an instance of Min-VCC, an instance $(T, \mathcal{M})$ of MinADD is constructed as follows. $T=\left(V_{T}, E_{T}\right)$ is defined as follows (see Fig. 2):

- $V_{T}=\left\{l_{i}, a_{i, 1}, a_{i, 2}: 1 \leq i \leq n\right\} \cup\left\{e_{i, j}: 1 \leq i \leq n \wedge 1 \leq j \leq 3\right\} ;$

$-E_{T}=\left\{\left\{l_{i}, l_{i+1}\right\}: 1 \leq i<n\right\} \cup\left\{\left\{l_{i}, a_{i, 1}\right\},\left\{a_{i, 1}, a_{i, 2}\right\}: 1 \leq i \leq\right.$ $n\} \cup\left\{\left\{a_{i, 2}, e_{i, j}\right\}: 1 \leq i \leq n \wedge 1 \leq j \leq 3\right\}$.

\section{$T$}

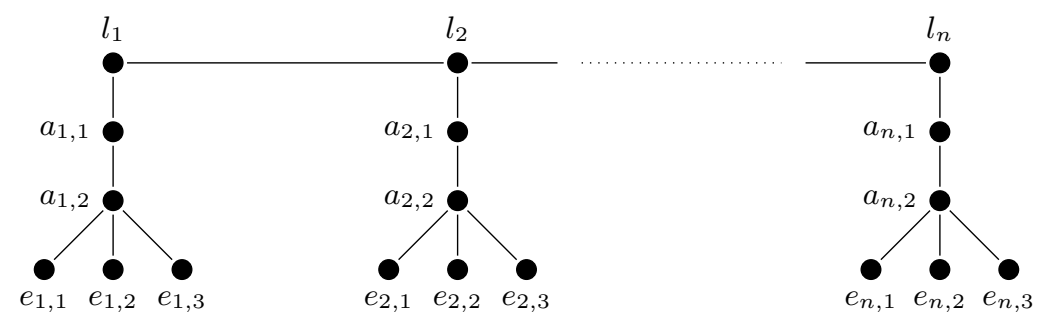

Fig. 2. Illustration of the reduction from Min-VCC to Min-ADD.

Each vertex $l_{i}, 1 \leq i \leq n$, is assigned a unique color $c\left(l_{i}\right), 1 \leq i \leq n$. The two vertices $a_{i, 1}, a_{i, 2}, 1 \leq i \leq n$, are assigned the same color $c\left(v_{i}\right)$. Finally, each vertex $e_{i, x}$ in $V_{T}, 1 \leq i \leq n$ and $1 \leq x \leq 3$, associated to an edge $e_{i, j}=\left\{v_{i}, v_{j}\right\}$ in $E$, is assigned color $c\left(e_{i, j}\right) . \mathcal{M}$ is a set of colors defined as follows: $\mathcal{M}=\left\{c\left(l_{i}\right): 1 \leq i \leq n\right\} \cup\left\{c\left(v_{i}\right): 1 \leq i \leq n\right\} \cup\left\{c\left(e_{i, j}\right): e_{i, j} \in E\right\}$.

It can be proved that starting from a vertex cover $V^{\prime} \subseteq V$ of $G$, we can compute in polynomial time a solution $V_{T^{\prime}}$ of MiN-ADD such that $C\left(V_{T^{\prime}}\right) \supseteq \mathcal{M}$ and $\left|C\left(V_{T^{\prime}}\right)\right| \leq|\mathcal{M}|+\left|V^{\prime}\right|$. Conversely, starting from a solution $V_{T^{\prime}}$ of Min-ADD such that $C\left(V_{T^{\prime}}\right) \supseteq \mathcal{M}$ and $\left|C\left(V_{T^{\prime}}\right)\right| \leq|\mathcal{M}|+p$, we can compute a vertex cover $V^{\prime}$ of $G$ such that $\left|V^{\prime}\right| \leq p$.

\section{Parameterized Complexity of Min-Substitute}

In this section, we discuss the parameterized complexity of Min-Substitute, when parameterized by $|\mathcal{M}|$. We recall that Min-Substitute is not in FPT, as discussed in Section 2, when parameterized by the the size of the solution (i.e., the number of substituted colors).

Let us first consider the case where the motif $\mathcal{M}$ is colorful (i.e., $\mathcal{M}$ is a set). The algorithm is based on dynamic programming. Let $(G=(V, E), \mathcal{M})$ be an instance of Min-SubstituTE. Instead of computing directly a solution for MinSubstitute, we compute a solution for a slightly different problem, where we visit the vertices of a connected component of $G$, allowing to visit some vertices 
more than once. Let $v$ be a vertex of the input graph $G$, let $C \subseteq \mathcal{M}$ be a subset of colors, let $k$ be the number of vertices of the solution of Min-Substitute we are looking for, and define $S[v, C, k]$ as the minimum value $z$ required by a visit of a connected set $V_{T}$ of vertices of $G$ such that:

1. $v \in V_{T}$

2. exactly $k$ visits of vertices in $V_{T}$ are done;

3. $C\left(V_{T}\right)$ matches $q$ colors of $C$, where $z=k-q$.

Notice that a vertex of $V_{T}$ may be visited more than once, while the overall number of visits must be $k$. Now, let us define the dynamic programming recurrence to compute $S[v, C, k]$.

$$
S[v, C, k]=\min _{C^{\prime} \subseteq C, u \in N(v), k_{1}+k_{2}=k}\left\{S\left[v, C^{\prime}, k_{1}\right]+S\left[u, C \backslash C^{\prime}, k_{2}\right]\right\} .
$$

For the base cases: $S\left[u, C^{\prime}, 1\right]=0$, when $c(u) \in C^{\prime}$, for each $C^{\prime} \subseteq C$ and $u \in V$, and $S\left[u, C^{\prime}, 1\right]=1$ when $c(u) \notin C^{\prime}$. Now, let us prove the correctness of Recurrence (1).

Lemma 2. Let $(G, \mathcal{M})$ be an instance of Min-Substitute, let $v$ be a vertex of $G$, and let $C$ be a subset of $\mathcal{M}$. There is a visit of a connected vertex set $V_{T}$ of $G$, such that $v \in V_{T}$, the vertices of $V_{T}$ are visited $k$ times, and $C\left(V_{T}\right)$ matches $q$ colors of $C$, iff there exists an entry $S[v, C, k]=z$, where $z=k-q$.

An optimal solution for the Min-Substitute problem can be found as follows. We look for the minimal value $z$ in the entries $S[v, \mathcal{M},|\mathcal{M}|]$, with $v \in V$. Notice that this value may be associated to a visit of a connected vertex set $V_{T}$, where some of the vertices may be visited repeatedly. Each repeated visit of a vertex represents a color to be substituted, since $\mathcal{M}$ is colorful. It follows that we can compute a feasible solution for Min-Substitute by replacing these repeated visits by some connected components adjacent to $V_{T}$ without increasing the number of substitutions.

The time complexity of the algorithm is $O^{*}\left(3^{|\mathcal{M}|}\right)$, as we have to consider all possible subsets $C \subseteq \mathcal{M}$ and for each subset $C$ we have to consider all possibile bipartitions of $C$. Indeed, there are $O\left(3^{|\mathcal{M}|}\right)$ possible bipartitions of all possible subsets $C$ of $\mathcal{M}$. In order to extend the results to a multiset, we apply the recoloring technique described in [4]. Combining Lemma 2 with Lemma 1, and we get that Min-Substitute, parameterized by $|\mathcal{M}|$, can be solved in time

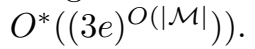

\section{Parameterized Complexity of CGM}

In this section, we consider the parameterized complexity of CGM, where the parameter is the number $k$ of optional occurrences $C_{o}$, that is $k=\left|C_{o}\right|$, where $C_{o}=\mathcal{M} \backslash C\left(V_{M}\right)$. First, in Section 5.1, we show that CGM is fixed-parameter tractable, when the input graph is of bounded treewidth ; then, in Section 5.2, we prove that CGM is W[2]-hard, even when the input graph is of diameter 2. 


\subsection{An FPT Algorithm for Graphs of Bounded Treewidth}

Here, we describe a fixed-parameter algorithm for CGM for graphs of bounded treewidth. Let $\left(G=(V, E), \mathcal{M}, V_{M}\right)$ be an instance of CGM, and let us first consider the case where the motif $\mathcal{M}$ is colorful.

Denote by $k$ the number of optional occurrences and by $\delta$ the treewidth of graph $G$. The algorithm is based on a nice tree decomposition of $G$ (see Section 2 for the definition of nice tree decomposition of a graph). We also consider a slightly more general problem, where instead of requiring that an occurrence of a motif consists of a single connected component, we may have an occurrence consisting of at most $\delta+1$ connected components, where the different connected components are induced by a partition of a bag $X_{i}$ of the nice tree decomposition. Given a vertex $i$ of the tree decomposition of $G$, denote by $T[i]$ the subtree of the nice tree decomposition rooted at $i$ and let $V(T[i])=\left\{u \in X_{j}: j \in T[i]\right\}$.

Now, consider a set $X_{i}, 1 \leq i \leq p$, of the nice tree decomposition $\left\langle\left\{X_{i}, i \in\right.\right.$ $\{1, \ldots, p\}\}, T\rangle$. From the definition of treewidth, it follows that $\left|X_{i}\right| \leq \delta+1$. Now, let us define a mapping function $f_{i}$ associated to the vertices of $X_{i}$, as follows.

Definition 1. Let $X_{i}$ be a bag of the nice tree decomposition of $G$. A mapping function $f_{i}$ from $X_{i}$ to $\{0,1, \ldots, \delta+1\}$ is feasible when

1. $f_{i}(v) \neq 0$ for each mandatory vertex $v$ in $X_{i}$;

2. for each pair of vertices $u, v \in X_{i}$ such that $c(u)=c(v)$, then $f_{i}(u)=0$ or $f_{i}(v)=0$

3. define $X_{i}^{l}=\left\{v \in X_{i}: f\left(v_{i}\right)=l\right\}, l \in\{1, \ldots, \delta+1\}$, and $X_{i}^{\prime}=\cup_{l} X_{i}^{l}$, then $X_{i}^{l}$ is a maximal connected component of $G\left[X_{i}^{\prime}\right]$.

A feasible mapping $f_{i}$ represents a partition of a subset $X_{i}^{\prime} \subseteq X_{i}$ in at most $\delta+1$ connected components, where $f_{i}(v)=p \neq 0$ implies that $v$ belongs to the $p$-th connected component, while $f_{i}(v)=0$ implies that $v$ does not belong to $X_{i}^{\prime}$.

Definition 2. Let $W$ be a set of vertices of $V(T[i])$, consisting of the connected components $W_{1}, W_{2}, \ldots, W_{z}$. Let $f_{i}$ be a feasible mapping of $X_{i}$ in $\{0,1, \ldots \delta+1\}$, then $W$ is mapped (or partitioned) according to $f_{i}$ if:

1. for each $p, 1 \leq p \leq z, W_{p} \cap X_{i} \neq \emptyset$, and there exists exactly one $l, 1 \leq l \leq$ $\delta+1$, such that $W_{p} \cap X_{i}=X_{i}^{l}$

2. for each $l, 1 \leq l \leq \delta+1$, such that $X_{i}^{l} \neq \emptyset$, there exists exactly one $p$, $1 \leq p \leq z$, such that $X_{i}^{l}=W_{p} \cap X_{i}$.

Notice that by Definition 2, if a vertex $u$ of $W$ is not in $X_{i}$, then there exists a vertex $v$ in $W \cap X_{i}$ such that $v$ and $u$ are in the same connected component $W_{x}$ of $W, v$ is assigned some label $l \neq 0$, and all the vertices of $W_{x} \cap X_{i}$ are assigned the same label $l$.

Given two sets $X_{i}$ and $X_{j}$ of a nice tree decomposition, and two feasible mappings $f_{i}: X_{i} \rightarrow\{0, \ldots, \delta+1\}$ and $f_{j}: X_{j} \rightarrow\{0, \ldots, \delta+1\}$, then $f_{i}$ and $f_{j}$ are consistent if, for each $v \in X_{i} \cap X_{j}, f_{i}(v)=f_{j}(v)$. 
Let $i$ be a vertex of the nice tree decomposition, with exactly one child $j$, such that $\left|X_{i}\right|=\left|X_{j}\right|+1$ and $X_{j} \subset X_{i}$, with $v \in X_{i} \backslash X_{j}$. Then, a feasible mapping $f_{i}$ is an extension of a feasible mapping $f_{j}$, when either:

1. $f_{i}(v)=0$; or

2. $f_{i}(v)=l, l \in\{1, \ldots, \delta+1\}, f_{i}(u) \neq l$ for each $u \in X_{i} \cap X_{j}$, and $f_{i}, f_{j}$ are consistent; or

3. there exists a value $l \in\{1, \ldots, \delta+1\}$ such that

(a) $f_{i}(v)=l$

(b) if $f_{j}(z)=0$, then $f_{i}(z)=0$, for $z \in X_{i} \cap X_{j}$;

(c) if $f_{i}(z) \neq f_{j}(z)$, for $z \in X_{i}$ and $f_{j}(z) \neq 0$, then $f_{i}(z)=l$.

Given a feasible mapping $f_{i}$ of $X_{i}$ in $\{0,1, \ldots \delta+1\}$, define $c\left(X_{i}, f_{i}\right)=\{c \in$ $\left.C_{o}: \exists v \in X_{i}, c(v)=c \wedge f_{i}(v) \neq 0\right\}$.

Let us define the value $S\left[i, f_{i}, C^{\prime}\right]$, where $i$ is a vertex of the nice tree decomposition of $G, f_{i}$ is a feasible mapping function of the set $X_{i}$ in $\{0,1, \ldots \delta+1\}$ and $C^{\prime} \subseteq C_{o}$ be a subset of the set of optional occurrences. $S\left[i, f_{i}, C^{\prime}\right]=1$ when there exists a set $W$ of vertices in the nice tree decomposition rooted at $i$, such that the vertices of $W$ can be partitioned according to $f_{i}$, each mandatory vertex of $T[i]$ is in $W$, and the set of optional occurrences of $c(W)$ is $C^{\prime}$; else $S\left[i, f_{i}, C^{\prime}\right]=0$. Next, we describe how to compute $S\left[i, f_{i}, C^{\prime}\right]$ by dynamic programming, depending on the three different cases of a nice tree decomposition.

Case 1) Assume that vertex $i$ has two children $j$ and $k$ (recall that $X_{i}=X_{j}=$ $X_{k}$ ), then

$$
S\left[i, f_{i}, C^{\prime}\right]=\bigvee_{f_{j}, f_{k}, C_{j}, C_{k}} S\left[j, f_{j}, C_{j}\right] \wedge S\left[k, f_{k}, C_{k}\right],
$$

where $f_{i}, f_{j}, f_{k}$ are all feasible and consistent, $C^{\prime}=\left(C_{j} \cup C_{k}\right)$ and $c\left(X_{i}, f_{i}\right)=C_{j} \cap C_{k}$.

Case 2) Assume that $i$ has exactly one child $j$, such that $X_{i}=X_{j} \cup\{v\}$, then

$$
S\left[i, f_{i}, C^{\prime}\right]=\bigvee_{f_{j}, C_{j}} S\left[j, f_{j}, C_{j}\right],
$$

where $f_{i}$ and $f_{j}$ are feasible, $f_{i}$ is an extension of $f_{j}, C^{\prime}=C_{j} \cup\{c(v)\}$ and $c(v) \notin C_{j}$, when $f_{i}(v) \neq 0$ and $v \notin V_{M}$, and $C^{\prime}=C_{j}$ when $f_{i}(v)=0$ or $v \in V_{M}$.

Case 3) Assume that $X_{i}$ has exactly one child $X_{j}$, such that $X_{i}=X_{j} \backslash\{v\}$, then

$$
S\left[i, f_{i}, C^{\prime}\right]=\bigvee_{f_{j}} S\left[j, f_{j}, C^{\prime}\right],
$$

where $f_{i}$ and $f_{j}$ are feasible and consistent, and there is a vertex $z \in X_{i} \cap X_{j}$, such that $f_{j}(z)=f_{j}(v)$, with $v \in X_{j} \backslash X_{i}$, when $f_{j}(v) \neq 0$. 
For the base cases (when $X_{i}$ is a leaf of the nice tree decomposition), define $S\left[i, f_{i}, C^{\prime}\right]=1$ when there is a partition of the vertices of $X_{i}$ according to the feasible function $f_{i}$, and $c\left(X_{i}, f_{i}\right)=C^{\prime}$; else $S\left[i, f_{i}, C^{\prime}\right]=0$.

First, we prove the correctness of the above recurrences, then we discuss the time complexity of the algorithm.

Lemma 3. Let $f_{i}$ be a feasible mapping function of $X_{i}$, and let $W$ be a set of vertices in $V(T[i])$, such that $W$ contains all the mandatory vertices in $V(T[i])$, $W$ can be mapped according to $f_{i}$ and $C^{\prime}$ is the set of optional occurrences in $c(W)$. Then $S\left[i, f_{i}, C^{\prime}\right]=1$.

Lemma 4. Let $S\left[i, f_{i}, C^{\prime}\right]=1$ for a feasible mapping function $f_{i}$ of $X_{i}$ in $\{0,1, \ldots, \delta+1\}$, then there exists a set $W$ of vertices in $V(T[i])$ such that the set of optional occurrences in $c(W)$ is $C^{\prime}, W$ contains all the mandatory vertices in $V(T[i])$ and the vertices of $W$ can be mapped according to $f_{i}$.

Theorem 3 shows how the values $S\left[i, f_{i}, C^{\prime}\right]$ are used to compute the existence of a feasible solution for CGM.

Theorem 3. Let $\left(G=(V, E), \mathcal{M}, V_{M}\right)$ be an instance of the CGM problem. Then there is a solution $W$ of CGM over instance of $\left(G, \mathcal{M}, V_{M}\right)$ iff there is a vertex $i$ of the nice tree decomposition and a feasible function $f_{i}$ that maps $X_{i}$ in $\{0, x\}$, with $x \in\{1, \ldots, \delta+1\}$, such that $S\left[i, f_{i}, C_{o}\right]=1$ and such $V_{M} \subseteq V(T[i])$.

Proof. Assume that there is a vertex $i$ of the nice tree decomposition and a feasible function $f_{i}$ that maps $X_{i}$ in $\{0, x\}$, with $x \in\{1, \ldots, \delta+1\}$, such that $S\left[i, f_{i}, C_{o}\right]=1$ and all the mandatory vertices of $G$ are in $T[i]$. By Lemma 4 , it follows that there is a set of vertices $W$ in $V(T[i])$ that contains all the mandatory vertices of $G$, such that the set of optional occurrences in $c(W)$ is $C_{o}$ and such that the vertices of $W$ can be mapped according to $f_{i}$. Furthermore, notice that $W$ consists of a single connected component. Hence $W$ is a solution of CGM.

Consider the case where there is a solution $W$ of CGM over instance $(G=$ $\left.(V, E), \mathcal{M}, V_{M}\right)$. Consider a vertex $i$ of the tree decomposition of $G$ such that all the vertices of $W$ are contained in $V(T[i])$. By Lemma 3, it follows that $S\left[i, f_{i}, C_{o}\right]=1$ for some feasible function $f_{i}$ that maps $X_{i}$ in $\{0, x\}$, with $x \in$ $\{1, \ldots, \delta+1\}$.

Now, we discuss the time complexity of the above algorithm. Denote by $n$ the size of $V$. Given a vertex $i$ and the associated set $X_{i}$ of the nice tree decomposition, the number of possible mapping functions of $X_{i}$ into $\{0, \ldots, \delta+1\}$ is $O\left(\delta^{\delta}\right)$. The number of possible subsets $C^{\prime}$ is $O\left(2^{k}\right)$. Since the number of vertices of a nice tree decomposition is $O(n)$, it follows that we have $O\left(\delta^{\delta} n 2^{k}\right)$ entries $S\left[i, f_{i}, C\right]$. Given a mapping function $f_{i}$ of $X_{i}$ into $\{0, \ldots, \delta+1\}$, computing an entry $S\left[i, f_{i}, C\right]$, given the entries of the children (or the child) of $i$, requires time at most $O\left(\delta^{2 \delta} 2^{2 k}\right)$ (notice that the worst case occurs when $i$ has two children). Hence the total time complexity is $O\left(\delta^{3 \delta} n 2^{3 k}\right)$.

When a motif is a multiset of colors, we apply the recoloring technique presented in Lemma 1. As a consequence, CGM can be solved with error probability $\varepsilon$ in time $O\left(|\ln (\varepsilon)| \delta^{3 \delta} n 2^{4.4427 k}\right)$, for graphs of treewidth $\delta$. 


\subsection{Hardness of Parameterization}

The CGM problem parameterized by the number of optional occurrences is $W[2]$-hard, as stated in Section 2. Here we strengthen the result, showing that the problem is $W[2]$-hard even when the input graph is of diameter 2.

Theorem 4. The CGM problem, parameterized by the number of optional occurrences, is W[2]-hard, even when the input graph is of diameter 2.

Proof. (Sketch) We give a parameterized preserving reduction from Minimum Set Cover (Min-SC). Given a universe $U=\left\{u_{1}, \ldots, u_{n}\right\}$ and a collection of sets $\mathcal{S}=\left\{S_{1}, \ldots, S_{m}\right\}$ over $U$, CGM asks for a collection $\mathcal{S}^{\prime}$ of at most $k$ sets of $\mathcal{S}$, such that $\bigcup_{S_{i}^{\prime} \in \mathcal{S}^{\prime}} S_{i}^{\prime}=U$. Min-SC is known to be $W[2]$-hard [16]. Let $(U, \mathcal{S})$ be

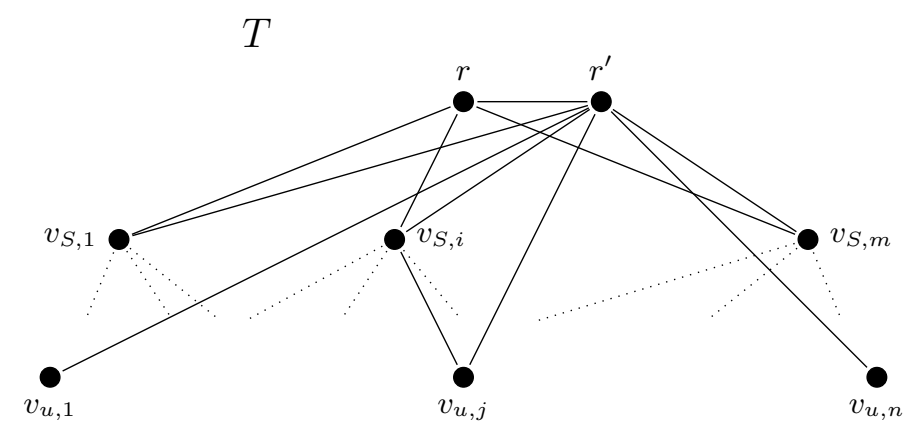

Fig. 3. Illustration of the reduction from Min-SC to CGM ; notice that element $u_{j} \in$ $S_{i}$.

an instance of Min-SC, we define a corresponding instance $\left(G=(V, E), \mathcal{M}, V_{M}\right)$ of the CGM problem (see Fig. 3). The graph $G$ of diameter 2 is defined as follows:

$$
\begin{aligned}
& -V=\{r\} \cup\left\{r^{\prime}\right\} \cup\left\{v_{S, i}: 1 \leq i \leq m\right\} \cup\left\{v_{u, j}: 1 \leq j \leq n\right\} ; \\
& -E=\left\{\left\{r, r^{\prime}\right\}\right\} \cup\left\{\left\{r, v_{S, i}\right\}: 1 \leq i \leq m\right\} \cup\left\{\left\{r^{\prime}, v_{S, i}\right\}: 1 \leq i \leq m\right\} \cup \\
& \quad\left\{\left\{v_{S, i}, v_{u, j}\right\}: 1 \leq i \leq m \wedge u_{j} \in S_{i}\right\} \cup\left\{\left\{r^{\prime}, v_{u, j}\right\}: 1 \leq j \leq n\right\} .
\end{aligned}
$$

Vertex $r$ and vertex $r^{\prime}$ are both colored by $c(r)$, vertex $v_{S, i}$ is colored by $c(S)$, $1 \leq i \leq m$, and vertex $v_{u, j}$ is colored by $c\left(u_{j}\right), 1 \leq j \leq n$. The motif $\mathcal{M}$ is a multiset containing one occurrence of color $c(r)$, one occurrence of each color $c\left(u_{j}\right), 1 \leq j \leq n$, and $k$ occurrences of color $c(S)$. Finally, $V_{M}=V \backslash$ $\left(\left\{v_{S, i}: 1 \leq i \leq m\right\} \cup\left\{r^{\prime}\right\}\right)$.

Then, it is possible to show that, given a solution of Min-SC of size at most $k$, we can compute in polynomial time a solution of CGM over instance $\left(G=(V, E), \mathcal{M}, V_{M}\right)$. Similarly, it is possible to show that given an occurrence 
$V_{T}$ of motif $\mathcal{M}$ in $G$, we can compute in polynomial time a solution of MiN$\mathrm{SC}$ of size at most $k$. By construction, a solution of CGM over instance $(G=$ $\left.(V, E), \mathcal{M}, V_{M}\right)$ contains exactly $k$ optional occurrences. Hence the reduction is parameter preserving, thus implying that CGM is $W[2]$-hard.

\section{References}

1. Ambalath, A. M. , Balasundaram, R., Chintan, R., Koppula, V., Misra, N., Philip, G., Ramanujan M.S.: On the Kernelization Complexity of Colorful Motifs. In: Raman, V., Saurabh, S. (eds.) IPEC 2010. LNCS, vol. 6478, pp. 14-25. Springer, Heidelberg (2010)

2. Alimonti, P., Kann, V.: Some APX-Completeness Results for Cubic Graphs. Theor. Comput. Sci. 237(1-2), 123 - 134 (2000)

3. Alon, N., Yuster, R., Zwick, U.: Color Coding. Journal of the ACM 42(4), 844-856 (1995)

4. Betzler, N., Fellows, M.R., Komusiewicz, C., Niedermeier, R.: Parameterized Algorithms and Hardness Results for Some Graph Motif Problems. In: Ferragina, P., Landau, G. (eds.) CPM 2008. LNCS, vol. 5029, pp. 31-43. Springer, Heidelberg (2008)

5. Bruckner, S., Hüffner, F., Karp, R.M., Sharan, R., Shamir, R.: Topology-Free Querying of Protein Interaction Networks. In: Batzoglou, S. (ed.) RECOMB 2009. LNCS, vol. 5541, pp. 74-89. Springer, Heidelberg (2009)

6. Cesati, M.: Compendium of parameterized problems, http://bravo.ce.uniroma2.it/home/cesati/research/compendium.pdf

7. Dondi, R., Fertin, G., Vialette, S.: Weak Pattern Matching in Colored Graphs: Minimizing the Number of Connected Components. In: Italiano, G. F., Moggi, E., Laura, L. (eds.) ICTCS 2007, pp. 27-38. World Scientific, Singapore (2007)

8. Dondi, R., Fertin, G., Vialette, S.: Maximum Motif in Vertex-Colored Graphs. In: Kucherov, G., Ukkonen, E. (eds.) CPM 2009. LNCS, vol. 5577, pp. 221-235. Springer, Heidelberg (2009)

9. Downey, R., Fellows, M.: Parameterized Complexity. Springer, Heidelberg (1999)

10. Fellows, M., Fertin, G., Hermelin, D., Vialette S.: Sharp Tractability Borderlines for Finding Connected Motifs in Vertex-Colored Graphs. In: Arge, L., Cachin, C., Jurdzinski, T., Tarlecki, A. (eds.) ICALP 2007. LNCS, vol. 4596, pp. 340-351. Springer, Heidelberg (2007)

11. Guillemot, S., Sikora, F.: Finding and Counting Vertex-Colored Subtrees. In: Hlinený, P., Kucera, A. (eds.) MFCS 2010. LNCS, vol. 6281, pp. 405-416. Springer, Heidelberg (2010)

12. Kelley, B. P., Sharan, R., Karp, R.M., Sittler, T., Root, D.E., Stockwell, B.R., Ideker, T., Conserved Pathways within Bacteria and Yeast as Revealed by Global Protein Network Alignment. Proc. Nat. Acad. Sci. 100(20), 11394-11399 (2003)

13. Koyutürk, M., Grama, A., Szpankowski, W.: Pairwise Local Alignment of Protein Interaction Networks Guided by Models of Evolution. In: Miyano, S., Mesirov, J.P., Kasif, S., Istrail, S., Pevzner, P.A., Waterman. M.S. (eds.) RECOMB 2005. LNCS, vol. 3500, pp. 48-65. Springer, Heidelberg (2005)

14. Lacroix, V., Fernandes, C.G., Sagot, M. F.: Motif Search in Graphs: Application to Metabolic Networks. IEEE/ACM Transactions on Computational Biology and Bioinformatics (TCBB) 3(4), 360-368 (2006) 
15. Niedermeier, R.: Invitation to Fixed-Parameter Algorithms. Oxford University Press, Oxford (2006)

16. Paz, A., Moran, S.: Non Deterministic Polynomial Optimization Problems and Their Approximations. Theor. Comput. Sci. 15, 251-277 (1981)

17. Scott, J., Ideker, T., Karp, R.M., Sharan, R.: Efficient Algorithms for Detecting Signaling Pathways in Protein Interaction Networks. Journal of Computational Biology 13, 133-144 (2006)

18. Sharan, R., Ideker, T., Kelley, B., Shamir, R., Karp, R. M.: Identification of Protein Complexes by Comparative Analysis of Yeast and Bacterial Protein Interaction Data. In: Bourne, P. E., Gusfield, D. (eds.) RECOMB 2004, pp. 282-289. ACM Press, New York (2004)

19. Sharan, R., Suthram, S., Kelley, R., Kuhn, T., McCuine, S., Uetz, P., Sittler, Karp, R.M., Ideker, T.: Conserved Patterns of Protein Interaction in Multiple Species. Proc. Nat. Acad. Sci. 102(6), 1974-1979 (2005) 